ESAIM: COCV 22 (2016) 1264-1281

DOI: $10.1051 / \mathrm{cocv} / 2016049$
ESAIM: Control, Optimisation and Calculus of Variations

www.esaim-cocv.org

\title{
LOCAL EXACT BILINEAR CONTROL OF THE SCHRÖDINGER EQUATION *
}

\author{
JEAN-PIERRE PUEL ${ }^{1}$
}

\begin{abstract}
We are going to prove the local exact bilinear controllability for a Schrödinger equation, set in a bounded regular domain, in a neighborhood of an eigenfunction corresponding to a simple eigenvalue in dimension $N \leq 3$. For a general domain we will require a non degeneracy condition of the normal derivative of the eigenfunction on a part $\Gamma_{0}$ of the boundary satisfying the Geometric Control Condition (see [G. Lebeau. J. Math. Pures Appl. 71 (1992) 267-291]) and for a rectangle when $N=2$ or an interval for $N=1$ no further condition. In the general case we will use real potentials concentrated in the neighborhood of $\Gamma_{0}$ and the linear controllability results with real and sufficiently regular controls.
\end{abstract}

Mathematics Subject Classification. 35B65, 35Q41.

Received June 6, 2016. Accepted June 7, 2016.

\section{INTRODUCTION}

We are interested in the exact controllability of a Schrödinger equation in a neighborhood of an eigenfunction, the control being the real potential in the Schrödinger equation. This is then a bilinear controllability problem.

The case of the equation set on the whole space $\mathbb{R}^{N}$ is interesting but we will not consider it in the present work.

More precisely, if $\Omega$ is a bounded regular open set of $\mathbb{R}^{N}$ with boundary $\Gamma$, we consider the Schrödinger equation for $T>0$

$$
\left\{\begin{array}{l}
i \frac{\partial y}{\partial t}+\Delta y+V y=0 \text { in } \Omega \times(0, T) \\
y=0 \text { on } \Gamma \times(0, T) \\
y(0)=y_{0} \text { in } \Omega
\end{array}\right.
$$

Here $V$ is the potential and $y_{0}$ is the initial data for which we will make precise assumptions later on.

We denote by $\left(\lambda_{k}\right)_{k=1, \ldots,+\infty}$ and $\left(\varphi_{k}\right)_{k=1, \ldots,+\infty}$ the eigenvalues and the corresponding normalized eigenfunctions of the Laplace operator with Dirichlet boundary conditions. The eigenvalues are real, we also take the eigenfunctions real, and we then have

$$
\left\{\begin{array}{l}
-\Delta \varphi_{k}=\lambda_{k} \varphi_{k} \text { in } \Omega \\
\varphi_{k}=0 \text { on } \Gamma \\
\int_{\Omega} \varphi_{k} \varphi_{j} \mathrm{~d} x=\delta_{k, j} \forall k, j=1, \ldots,+\infty
\end{array}\right.
$$

Notice that if $z \in \mathbb{C}, z \neq 0$, then $z \varphi_{k}$ is also an eigenfunction of the Laplace operator.

Keywords and phrases. Schrödinger equation, bilinear control.

* Dedicated to Jean-Michel Coron on the occasion of his 60th birthday. Avec mon admiration et mes meilleures amitiés.

1 Laboratoire de Mathématiques de Versailles, Université de Versailles St Quentin, 78035 Versailles cedex, France.

jppuel@math.uvsq.fr 
If we take the free Schrödinger equation (with zero potential) with initial value $\varphi_{k}$, then the corresponding solution $\tilde{\varphi}_{k}$ is given by

$$
\tilde{\varphi}_{k}(t)=\mathrm{e}^{-i \lambda_{k} t} \varphi_{k}
$$

We can now formulate our controllability problem.

Given $y_{0}$, can we find a real potential $V$ such that at time $T$ we have

$$
y(T)=\tilde{\varphi}_{k}(T)=\mathrm{e}^{-i \lambda_{k} T} \varphi_{k} ?
$$

Of course this formulation will be made precise by the choice of the functional setting (for $V$ and $y_{0}$ ) which will be discussed later on. But we will need that (1.1) has a unique solution in a reasonable sense. As $V$ will be taken real, equation (1.1) will preserve the $L^{2}$ norm, so that an immediate necessary condition is that

$$
\int_{\Omega}\left|y_{0}\right|^{2} \mathrm{~d} x=1
$$

Therefore $y_{0}$ will be taken on the sphere of radius 1 in $L^{2}(\Omega)$ which we denote by $S$.

Results on local bilinear controllability for the Schrödinger equation have been obtained in the 1-dimensional case by $[1,2]$. In these articles, the potentials are taken of the form

$$
V(x, t)=u(t) \mu(x)
$$

where $\mu$ is a prescribed profile which has to satisfy some conditions on the boundary in addition to a sufficient regularity. The actual control is the amplitude $u($.$) . In that case, we have to notice that if X$ is the space of values of $y(t)$ and if the product by $\mu$ is bounded from $X$ to $X$, due to an abstract result of [4], there is no hope to obtain a control $u \in L_{\mathrm{loc}}^{r}(0, T), r>1$. In [2], an important fact is to consider as functional setting the space

$$
H_{\Delta}(\Omega)=\left\{z \in H_{0}^{1}(\Omega), \Delta z \in H_{0}^{1}(\Omega)\right\} .
$$

Notice that $H_{\Delta}(\Omega) \subset H^{3}(\Omega) \cap H_{0}^{1}(\Omega)$ when $\Omega$ is regular enough. It can be shown (using for example the decomposition on the orthonormal basis of eigenfunctions for the Laplace operator) that we also have

$$
H_{\Delta}(\Omega)=D\left((-\Delta)^{\frac{3}{2}}\right)
$$

Then the profile $\mu$ is taken such that

$$
\forall z \in H_{\Delta}(\Omega), \mu z \in H^{3}(\Omega) \cap H_{0}^{1}(\Omega) .
$$

but in general

$$
\mu z \notin H_{\Delta}(\Omega) .
$$

Using a regularity result for the free Schrödinger equation (proved in the 1-dimensional case in [2] and in the general case in [10]) and taking $u \in L^{2}(0, T)$, they prove their result using the controllability of the linearized problem and an inverse mapping theorem.

Unfortunately, in dimension $N \geq 2$, the linearized problem is no longer controllable when the potential is taken of the form $V(x, t)=u(t) \mu(x)$, with $\mu$ prescribed, and we cannot apply the same argument. In dimension $N=2$, in [3] the authors obtain a local controllability result, independently from the present work, by considering the interesting case of potentials $V$ satisfying the Poisson equation

$$
\left\{\begin{array}{l}
-\Delta V(t)+V(t)=0 \text { in } \Omega \\
V(t)=g(t) \text { on } \Gamma
\end{array}\right.
$$


the actual control being here the boundary value $g($.$) . In addition to the restriction on the dimension, their$ result requires some technical conditions on the eigenfunctions.

Here we will consider the case of dimension $N \leq 3$ and potentials $V$ such that $V$ is real and, for the eigenfunction $\varphi_{k}$ around which we will be working, $V \in L^{2}(0, T ; E)$ where

$$
E=\left\{V \in H^{2}(\Omega), V \varphi_{k} \in H^{3}(\Omega) \cap H_{0}^{1}(\Omega)\right\} .
$$

In fact $V$ will be continuous in time with values in $E$.

We have to notice that, as the control $V$ will depend on both $t$ and $x$, the abstract result of [4] does not apply anymore but this will not be of any help for us.

\section{Statement of the Result And Strategy of PRoof}

\subsection{Linear boundary and internal exact controllability problem}

First of all we recall some well known results on the linear boundary and internal exact controllability for the free Schrödinger equation.

The boundary controllability problem can be expressed as follows, due to the reversibility of the equation. Given a subset $\Gamma_{0}$ of $\Gamma$, for any $y_{0} \in H^{-1}(\Omega)$, can we find $g \in L^{2}\left(0, T ; L^{2}\left(\Gamma_{0}\right)\right)$ such that the solution $y$ of

$$
\left\{\begin{array}{l}
i \frac{\partial y}{\partial t}+\Delta y=0 \text { in } \Omega \times(0, T), \\
y=g \text { on } \Gamma_{0} \times(0, T), \\
y=0 \text { on }\left(\Gamma \backslash \Gamma_{0}\right) \times(0, T), \\
y(0)=y_{0} \text { in } \Omega
\end{array}\right.
$$

satisfies

Taking the adjoint problem

$$
y(T)=0
$$

$$
\left\{\begin{array}{l}
i \frac{\partial \varphi}{\partial t}+\Delta \varphi=0 \text { in } \Omega \times(0, T), \\
\varphi=0 \text { on } \Gamma \times(0, T), \\
\varphi(0)=\varphi_{0} \text { in } \Omega,
\end{array}\right.
$$

it is now well known that the exact controllability problem is equivalent to the following boundary observability inequality (for a constant $C$ independent of the initial value $\varphi_{0}$ )

$$
\left\|\varphi_{0}\right\|_{H_{0}^{1}(\Omega)}^{2} \leq C \int_{\Gamma_{0} \times(0, T)}\left|\frac{\partial \varphi}{\partial \nu}\right|^{2} \mathrm{~d} \sigma \mathrm{d} t
$$

where $\nu$ is the outward pointing unit normal vector to $\Gamma$.

In [9] the inequality is proved using the multiplier method for any $T>0$ with the following assumption on $\Gamma_{0}$.

$$
\text { There exists } x_{0} \in \mathbb{R}^{N} \text { such that } \Gamma_{0}=\left\{x \in \Gamma,\left(x-x_{0}\right) . \nu>0\right\} .
$$

This result has been extended in [7] using micro local analysis arguments to the case where $\Gamma_{0}$ satisfies the so-called geometric control condition (GCC), saying roughly speaking that every ray of the geometrical optics (reflecting on the boundary) reaches $\Gamma_{0}$ at a non diffractive point in uniform time.

In dimension $N=2$, the case of a rectangle for $\Omega$ is very particular and it is shown in [11] that we can take for $\Gamma_{0}$ the union of intervals on $\Gamma$ as soon as we take at least one interval in each direction.

The case of internal (distributed) exact controllability problem can be written as follows. Let $\omega$ be a non empty open subset of $\Omega$ and $\mathbb{I}_{\omega}$ be the characteristic function of $\omega$. Let $y$ be the solution of the following Schrödinger equation.

$$
\left\{\begin{array}{l}
i \frac{\partial y}{\partial t}+\Delta y=h \cdot \mathbb{I}_{\omega} \text { in } \Omega \times(0, T) \\
y=0 \text { on } \Gamma \times(0, T), \\
y(0)=y_{0} \text { in } \Omega
\end{array}\right.
$$


For any $y_{0} \in L^{2}(\Omega)$, can we find a control $h \in L^{2}\left(0, T ; L^{2}(\omega)\right)$ such that we have

$$
y(T)=0 .
$$

Again considering the adjoint problem (2.3) this is equivalent to the following internal observability inequality (for a constant $C$ independent of $\varphi_{0}$ )

$$
\left|\varphi_{0}\right|_{L^{2}(\Omega)}^{2} \leq C \int_{\omega \times(0, T)}|\varphi|^{2} \mathrm{~d} x \mathrm{~d} t
$$

In ([9], Prop. 3.1), the author proves that when $\Gamma_{0}$ is such that (2.4) is satisfied, then (2.8) is true when $\omega=\omega_{\eta}$ for $\eta>0$ is the neighborhood of $\Gamma_{0}$ defined by

$$
\omega_{\eta}=\bigcup_{x \in \Gamma_{0}}(B(x ; \eta) \cap \Omega) .
$$

For the particular case of a rectangle for $\Omega$ in dimension $N=2$ it is proved in [6] that (2.8) is valid for any non empty open subset $\omega$ of $\Omega$. Of course this is a fortiori valid in dimension $N=1$. We are now ready to state our results.

\subsection{Statement of the results}

For simplicity of the statement we separate the most general case and the case of a rectangle $(N=2)$ or an interval $(N=1)$.

Theorem 2.1. We assume that $N \leq 3$, and that $\Omega$ is a non empty bounded open set of $\mathbb{R}^{N}$ of class $C^{3, \alpha}$ with $\alpha>0$. Let $\Gamma_{0}$ be a non empty open subset of the boundary $\Gamma$ such that the boundary observability inequality $(2.4)$ is valid, and let $\left(\lambda_{k}, \varphi_{k}\right)$ be an eigenpair for the Laplace operator with Dirichlet boundary conditions. We assume that

(H1) $\lambda_{k}$ is a simple eigenvalue.

(H2) $\left|\frac{\partial \varphi_{k}}{\partial \nu}\right|>0$ on $\bar{\Gamma}_{0}$.

Then there exists $\delta>0$ such that for every $y_{0} \in H_{\Delta}(\Omega) \cap S$ with $\left\|y_{0}-\varphi_{k}\right\|_{H_{\Delta}(\Omega)} \leq \delta$ (where $H_{\Delta}(\Omega)$ is defined by (1.6) and $S$ is the unit sphere in $\left.L^{2}(\Omega)\right)$, there exists a real potential $V \in C(0, T ; E)$ such that the corresponding solution $y$ of (1.1) satisfies

$$
y(T)=\mathrm{e}^{-i \lambda_{k} T} \varphi_{k} .
$$

Theorem 2.2. We assume that $N=2$ and $\Omega$ is a rectangle or $N=1$ and $\Omega$ is an interval. Let $\left(\lambda_{k}, \varphi_{k}\right)$ be an eigenpair for the Laplace operator with Dirichlet boundary conditions. We assume hypothesis (H1) in the case $N=2$ and no hypothesis if $N=1$ (hypothesis (H1) is automatically satisfied). Then there exists $\delta>0$ such that for every $y_{0} \in H_{\Delta}(\Omega) \cap S$ with $\left\|y_{0}-\varphi_{k}\right\|_{H_{\Delta}(\Omega)} \leq \delta$ (where $H_{\Delta}(\Omega)$ is defined by (1.6) and $S$ is the unit sphere in $\left.L^{2}(\Omega)\right)$, there exists a real potential $V \in C([0, T] ; E)$ such that the corresponding solution y of (1.1) satisfies

$$
y(T)=\mathrm{e}^{-i \lambda_{k} T} \varphi_{k} .
$$

\section{Remark 2.3.}

(1) In fact our proof will show that we can also reach at time $T$ any target in a small neighborhood of $\mathrm{e}^{-i \lambda_{k} T} \varphi_{k}$ in $H_{\Delta}(\Omega) \cap S$.

(2) Hypotheses (H1) and (H2) are also assumed in [3]. But in this work, the authors also require $N=2$ and some additional technical assumptions on the eigenfunctions (and therefore on the geometry?). Nevertheless, we use here part of their intermediate results.

(3) Hypotheses (H1) and (H2) are of course satisfied if we take the first eigenpair $\left(\lambda_{1}, \varphi_{1}\right)$ but there exist several geometries for which other values of $k$ can be considered.

(4) As will be explained in the next subsection, the control $V$ will be taken with support in a neighborhood of a part of the boundary satisfying the Geometric Control Condition. 


\subsection{Formal strategy of proof}

We will restrict ourselves to the case of Theorem 2.1. All the time in the sequel we will take real potentials $V$ with support concentrated in a neighborhood of $\Gamma_{0}$.

For $y_{0} \in H_{\Delta}(\Omega) \cap S$ and $V \in C([0, T] ; E)$ (with the above restriction) we consider the solution $y$ of

$$
\left\{\begin{array}{l}
i \frac{\partial y}{\partial t}+\Delta y+V y=0 \text { in } \Omega \times(0, T) \\
y=0 \text { on } \Gamma \times(0, T) \\
y(0)=y_{0} \text { in } \Omega
\end{array}\right.
$$

We have to show that this problem is well posed and we will actually prove that there exists a unique solution $y \in C\left([0 . T], H_{\Delta}(\Omega) \cap S\right)$.

Then we can define the mapping

$$
\left(y_{0}, V\right) \rightarrow \Lambda\left(y_{0}, V\right)=\left(y(T), y_{0}\right) \in\left(H_{\Delta}(\Omega) \cap S\right)^{2} .
$$

Of course we have

$$
\Lambda\left(\varphi_{k}, 0\right)=\left(\mathrm{e}^{-i \lambda_{k} T} \varphi_{k}, \varphi_{k}\right)
$$

It will be shown that this mapping is of class $C^{1}$. If we can show that the derivative of $\Lambda$ at the point $\left(\varphi_{k}, 0\right)$ has a right inverse, then using an inverse mapping theorem, we will obtain our result (and the claim in Rem. 2.3).

It will be shown that for $z_{0} \in H_{\Delta}(\Omega) \cap T S_{k}$ and $W \in C([0, T] ; E)$ (real and concentrated near $\Gamma_{0}$ ) with

$$
\begin{gathered}
T S_{k}=\left\{z \in L^{2}(\Omega), \Re\left(z, \varphi_{k}\right)_{L^{2}(\Omega)}=0\right\} \\
\Lambda^{\prime}\left(\varphi_{k} \cdot 0\right)\left[z_{0}, W\right]=\left(\tilde{z}(T), z_{0}\right)
\end{gathered}
$$

where

$$
\left\{\begin{array}{l}
i \frac{\partial \tilde{z}}{\partial t}+\Delta \tilde{z}+W \mathrm{e}^{-i \lambda_{k} t} \varphi_{k}=0 \text { in } \Omega \times(0, T) \\
\tilde{z}=0 \text { on } \Gamma \times(0, T) \\
\tilde{z}(0)=z_{0} \text { in } \Omega
\end{array}\right.
$$

Writing

$$
z=\mathrm{e}^{i \lambda_{k} t} \tilde{z}
$$

(2.15) can be rewritten as

$$
\left\{\begin{array}{l}
i \frac{\partial z}{\partial t}+\Delta z+\lambda_{k} z+W \varphi_{k}=0 \text { in } \Omega \times(0, T) \\
z=0 \text { on } \Gamma \times(0, T) \\
z(0)=z_{0} \text { in } \Omega
\end{array}\right.
$$

We will then have to show that given any $\left(z_{0}, z_{1}\right) \in\left(H_{\Delta}(\Omega) \cap T S_{k}\right)^{2}$, we can find (continuously) a real potential $W \in C([0 . T] ; E)$ (concentrated near $\left.\Gamma_{0}\right)$ such that the solution of $(2.16)$ verifies

$$
z(T)=z_{1} .
$$

Due to the reversibility of equation (2.16), it is immediate to see that without loss of generality, we can take $z_{1}=0$.

We will then have to show a null controllability result for (2.16) with real control of the form $W \varphi_{k}$ and also to show a suitable regularity property for this control. 


\section{Proof of the Results}

\subsection{Proof of Theorem 2.1}

First of all as already said, for $\epsilon>0$ we consider the open subset of $\Omega$ defined by

$$
\omega_{\epsilon}=\bigcup_{x \in \Gamma_{0}}(B(x, \epsilon) \cap \Omega) .
$$

Because of hypothesis (H2) and the fact that $\varphi_{k} \in C^{1}(\bar{\Omega})$, we know that there exists $\epsilon_{0}>0$ such that

$$
\varphi_{k} \neq 0 \text { in } \omega_{\epsilon_{0}} \text { and }\left|\frac{\partial \varphi_{k}}{\partial \nu}\right| \neq 0 \text { on } \partial \omega_{\epsilon_{0}} \cap \Gamma \text {. }
$$

We now take $\epsilon_{1}$ and $\epsilon$ such that $0<\epsilon_{1}<\epsilon<\epsilon_{0}$ and we define a cut-off function $\chi_{\omega_{\epsilon}} \in C_{0}^{\infty}\left(\omega_{\epsilon} \cup(\partial \omega \epsilon \cap \Gamma)\right)$ such that

$$
\left\{\begin{array}{l}
0 \leq \chi_{\omega_{\epsilon}} \leq 1 \\
\chi_{\omega_{\epsilon}} \geq \mathbb{I}_{\omega_{\epsilon_{1}}} .
\end{array}\right.
$$

For simplicity we now omit the subscript $\epsilon$ and we write $\omega=\omega_{\epsilon}$ and $\chi_{\omega}=\chi_{\omega_{\epsilon}}$.

Lemma 3.1. If $y \in H^{3}(\Omega) \cap H_{0}^{1}(\Omega)$ then $\chi_{\omega} \cdot \frac{y}{\varphi_{k}} \in H^{2}(\Omega)$.

Proof. In fact this result can be proved locally and it is enough to prove it in a (small) neighborhood still denoted by $\omega$ (even if it can be smaller than $\omega$ ) of any point of $\partial \omega \cap \Gamma$ and then we can take $\chi_{\omega}=1$. We can suppose, after translation and rotation, that this point is the origin and that the coordinates are $\left(x^{\prime}, x_{N}\right)$ where $x^{\prime}=\left(x_{1}, \ldots, x_{N-1}\right)$ are coordinates of the tangent plane at the origin and $x_{N}$ is the normal variable. Without loss of generality, we can suppose that in $\omega$ we have $\varphi_{k}\left(x^{\prime}, x_{N}\right)>0$ (and of course vanishes on the boundary). We can now take new coordinates $\left(x^{\prime}, \xi\right)$ where $\xi=\varphi_{k}\left(x^{\prime}, x_{N}\right)$. This gives a $C^{3}$ diffeomorphism and the (local) images of $\Gamma$ and $\omega$ can be taken as $\{\xi=0\}$ and $\left\{0<\xi<\frac{\epsilon}{2}\right\}$. We can write

$$
y\left(x^{\prime}, x_{N}\right)=\tilde{y}\left(x^{\prime}, \xi\right)=\int_{0}^{1} \frac{\mathrm{d}}{\mathrm{d} t} \tilde{y}\left(x^{\prime}, t \xi\right) \mathrm{d} t=\int_{0}^{1} \frac{\partial \tilde{y}}{\partial \xi}\left(x^{\prime}, t \xi\right) \xi \mathrm{d} t
$$

Therefore

$$
\frac{\tilde{y}\left(x^{\prime}, \xi\right)}{\xi}=\int_{0}^{1} \frac{\partial \tilde{y}}{\partial \xi}\left(x^{\prime}, t \xi\right) \mathrm{d} t \in H^{2}(\omega)
$$

Lemma 3.2. When $N \leq 3$, for any $y \in H_{\Delta}(\Omega)$ and $V \in E$, then $\chi_{\omega} . V y \in H^{3}(\Omega) \cap H_{0}^{1}(\Omega)$ and the mapping

$$
(y, V) \in H_{\Delta}(\Omega) \times E \rightarrow \chi_{\omega} . V y \in H^{3}(\Omega) \cap H_{0}^{1}(\Omega)
$$

is bilinear continuous.

Proof. We have (recall that as $N \leq 3, H^{2}(\Omega) \subset L^{\infty}(\Omega)$ and $H^{1}(\Omega) \subset L^{6}(\Omega)$ )

$$
\begin{aligned}
\chi_{\omega} . V y \in L^{2}(\Omega), \frac{\partial}{\partial x_{i}}\left(\chi_{\omega} V y\right)= & \chi_{\omega}\left(V \frac{\partial y}{\partial x_{i}}+\frac{\partial V}{\partial x_{i}} y\right)+\frac{\partial \chi_{\omega}}{\partial x_{i}} V y \in L^{2}(\Omega) . \\
\frac{\partial^{2}}{\partial x_{i} \partial x_{j}}\left(\chi_{\omega} V y\right)= & \chi_{\omega}\left(V \frac{\partial^{2} y}{\partial x_{i} \partial x_{j}}+\frac{\partial V}{\partial x_{j}} \frac{\partial y}{\partial x_{i}}+\frac{\partial V}{\partial x_{i}} \frac{\partial y}{\partial x_{j}}+\frac{\partial^{2} V}{\partial x_{i} \partial x_{j}} y\right) \\
& +\frac{\partial \chi_{\omega}}{\partial x_{j}} \frac{\partial V y}{\partial x_{i}}+\frac{\partial \chi_{\omega}}{\partial x_{i}} \frac{\partial V y}{\partial x_{j}}+\frac{\partial^{2} \chi_{\omega}}{\partial x_{i} \partial x_{j}} V y \in L^{2}(\Omega)
\end{aligned}
$$

Let us show that $\frac{\partial^{3}}{\partial x_{i} \partial x_{j} \partial x_{l}}\left(\chi_{\omega} V y\right) \in L^{2}(\Omega)$. 
The terms involving derivatives of $\chi_{\omega}$ do not make any problem. We only have to treat the terms in factor of $\chi_{\omega}$.

$$
\chi_{\omega} \frac{\partial}{\partial x_{l}}\left(V \frac{\partial^{2} y}{\partial x_{i} \partial x_{j}}\right)=\chi_{\omega} \frac{\partial V}{\partial x_{l}} \frac{\partial^{2} y}{\partial x_{i} \partial x_{j}}+\chi_{\omega} V \frac{\partial^{3} y}{\partial x_{i} \partial x_{j} \partial x_{l}}
$$

As

$$
\frac{\partial V}{\partial x_{l}} \in H^{1}(\Omega) \subset L^{6}(\Omega) \text { and } \frac{\partial^{2} y}{\partial x_{i} \partial x_{j}} \in H^{1}(\Omega) \subset L^{6}(\Omega)
$$

we have

$$
\chi_{\omega} \frac{\partial V}{\partial x_{l}} \frac{\partial^{2} y}{\partial x_{i} \partial x_{j}} \in L^{2}(\Omega)
$$

As

$$
V \in L^{\infty}(\Omega) \text { and } \frac{\partial^{3} y}{\partial x_{i} \partial x_{j} \partial x_{l}} \in L^{2}(\Omega)
$$

we have

$$
\chi_{\omega} V \frac{\partial^{3} y}{\partial x_{i} \partial x_{j} \partial x_{l}} \in L^{2}(\Omega)
$$

and therefore

$$
\chi_{\omega} \frac{\partial}{\partial x_{l}}\left(V \frac{\partial^{2} y}{\partial x_{i} \partial x_{j}}\right) \in L^{2}(\Omega)
$$

In the same way we show that

$$
\chi_{\omega} \frac{\partial}{\partial x_{l}}\left(\frac{\partial V}{\partial x_{j}} \frac{\partial y}{\partial x_{i}}+\frac{\partial V}{\partial x_{i}} \frac{\partial y}{\partial x_{j}}\right) \in L^{2}(\Omega) .
$$

Now we can write

$$
\chi_{\omega} \frac{\partial^{2} V}{\partial x_{i} \partial x_{j}} y=\frac{\partial^{2} V}{\partial x_{i} \partial x_{j}} \varphi_{k} \chi_{\omega} \frac{y}{\varphi_{k}}
$$

We know that

$$
\chi_{\omega} \frac{y}{\varphi_{k}} \in H^{2}(\Omega) .
$$

Let us show that

$$
\frac{\partial^{2} V}{\partial x_{i} \partial x_{j}} \varphi_{k} \in H^{1}(\Omega)
$$

which will end the proof as we have already seen that a product of a function in $H^{2}(\Omega)$ by a function in $H^{1}(\Omega)$ is element of $H^{1}(\Omega)$. We can write

$$
\frac{\partial^{2} V}{\partial x_{i} \partial x_{j}} \varphi_{k}=\frac{\partial^{2}}{\partial x_{i} \partial x_{j}}\left(V \varphi_{k}\right)-\frac{\partial V}{\partial x_{i}} \frac{\partial \varphi_{k}}{\partial x_{j}}-\frac{\partial V}{\partial x_{j}} \frac{\partial \varphi_{k}}{\partial x_{i}}-V \frac{\partial^{2} \varphi_{k}}{\partial x_{i} \partial x_{j}}
$$

and in the right hand side, each term belongs to $H^{1}(\Omega)$ so that

$$
\frac{\partial^{2} V}{\partial x_{i} \partial x_{j}} \varphi_{k} \in H^{1}(\Omega) \text {. }
$$

This proves that

$$
V y \in H^{3}(\Omega)
$$

and as $y$ vanishes on the boundary $\Gamma$ this shows that

$$
V y \in H^{3}(\Omega) \cap H_{0}^{1}(\Omega) .
$$


The above proof shows in fact that the mapping

$$
(y, V) \in H_{\Delta}(\Omega) \times E \rightarrow \chi_{\omega} \cdot V y \in H^{3}(\Omega) \cap H_{0}^{1}(\Omega)
$$

is bilinear continuous and this finishes the proof of Lemma 3.2 .

From the above lemma we immediately deduce that the mapping

$$
(y, V) \in C\left([0, T] ; H_{\Delta}(\Omega)\right) \times C([0, T] ; E) \rightarrow \chi_{\omega} V y \in C\left([0, T] ; H^{3}(\Omega) \cap H_{0}^{1}(\Omega)\right)
$$

is bilinear continuous.

Lemma 3.3. For any $y_{0} \in H_{\Delta}(\Omega) \cap S$ and $V \in C([0, T] ; E)$, with $V$ real, there exists a unique solution $y \in C\left([0, T] ; H_{\Delta}(\Omega) \cap S\right)$ to the Schrödinger equation

$$
\left\{\begin{array}{l}
i \frac{\partial y}{\partial t}+\Delta y+\chi_{\omega} V y=0 \text { in } \Omega \times(0, T) \\
y=0 \text { on } \Gamma \times(0, T) \\
y(0)=y_{0} i n \Omega
\end{array}\right.
$$

Proof. Let us take $\tilde{y} \in C\left([0, T] ; H_{\Delta}(\Omega)\right)$. We know that the product $\chi_{\omega} V \tilde{y} \in C\left([0, T] ; H^{3}(\Omega) \cap H_{0}^{1}(\Omega)\right)$ and we can define $z$ as the solution of

$$
\left\{\begin{array}{l}
i \frac{\partial z}{\partial t}+\Delta z+\chi_{\omega} V \tilde{y}=0 \text { in } \Omega \times(0, T) \\
z=0 \text { on } \Gamma \times(0, T) \\
z(0)=y_{0} \text { in } \Omega
\end{array}\right.
$$

From the regularity result of $[10]$ we have in fact $z \in C\left([0, T] ; H_{\Delta}(\Omega)\right)$ and there exists a constant $C$ independent of the initial value $y_{0}$ and of $T \leq 1$ such that

$$
\begin{array}{r}
\|z\|_{C([0, T] ; H \Delta(\Omega))} \leq C\left(\left\|y_{0}\right\|_{H_{\Delta}(\Omega)}+\|V \tilde{y}\|_{L^{2}\left(0, T ; H^{3}(\Omega) \cap H_{0}^{1}(\Omega)\right)}\right) \\
\leq C\left(\left\|y_{0}\right\|_{H_{\Delta}(\Omega)}+\sqrt{T}\|V\|_{C([0, T] ; E)}\|\tilde{y}\|_{C([0, T] ; H \Delta(\Omega))}\right) .
\end{array}
$$

Notice that the assumption $V \in C([0, T] ; E)$ is used here to obtain a positive power of $T$ in the second term of the right hand side. Of course we could have taken $V \in L^{p}(0, T ; E)$ with $p>2$.

Then, using a classical fixed point method for $T$ small but independent of the initial value, we obtain existence and uniqueness for the solution $y$ of $(3.3)$ in $C\left([0, T] ; H_{\Delta}(\Omega)\right)$, first for small $T$ (but independent of the initial value), then by simple iterations for any $T>0$ with

$$
y \in C\left([0, T] ; H_{\Delta}(\Omega)\right) .
$$

If $y_{0} \in S$, then as $V$ is real, the equation preserves the $L^{2}$ norm and $y(t) \in S$ for $t \in[0, T]$. This finishes the proof of Lemma 3.3.

We can now define the mapping $\Lambda$ by

$$
\Lambda\left(y_{0}, V\right)=\left(y(T), y_{0}\right)
$$

and $\Lambda$ maps continuously $H_{\Delta}(\Omega) \cap S \times C([0, T] ; E)$ into $\left(H_{\Delta}(\Omega) \cap S\right)^{2}$. We have

$$
\Lambda\left(\varphi_{k}, 0\right)=\left(\tilde{\varphi}_{k}(T), \varphi_{k}\right)=\left(\mathrm{e}^{-i \lambda_{k} T} \varphi_{k}, \varphi_{k}\right) .
$$


Lemma 3.4. The mapping $\Lambda$ is differentiable at $\left(\varphi_{k}, 0\right)$ and for any $z_{0} \in H_{\Delta}(\Omega) \cap T S_{k}$ and $W \in C([0, T] ; E)$ we have

$$
\Lambda^{\prime}\left(\varphi_{k}, 0\right)\left[z_{0}, W\right]=\left(\tilde{z}(T), z_{0}\right)
$$

where

$$
\left\{\begin{array}{l}
i \frac{\partial \tilde{z}}{\partial t}+\Delta \tilde{z}+\chi_{\omega} W \tilde{\varphi}_{k}=0 \text { in } \Omega \times(0, T), \\
\tilde{z}=0 \text { on } \Gamma \times(0, T), \\
\tilde{z}(0)=z_{0} \text { in } \Omega
\end{array}\right.
$$

Moreover, $\tilde{z}(T) \in H_{\Delta}(\Omega)$ and $\Re\left(\tilde{z}(T), \tilde{\varphi}_{k}(T)\right)_{L^{2}(\Omega)}=0$.

Proof. For $y_{0} \in H_{\Delta}(\Omega) \cap S$ we denote by $P_{k} y_{0}$ the projection of $y_{0}-\varphi_{k}$ on $H_{\Delta}(\Omega) \cap T S_{k}$ and we have to write the expression of

$$
\Lambda\left(y_{0}, V\right)-\Lambda\left(\varphi_{k}, 0\right)-\Lambda^{\prime}\left(\varphi_{k}, 0\right)\left[P_{k} y_{0}, V\right] .
$$

We still write $\tilde{z}$ for the solution of (3.7) with $z_{0}=P_{k} y_{0}$. If

$$
w=y-\tilde{\varphi}_{k}-\tilde{z}
$$

we have

$$
\left\{\begin{array}{l}
i \frac{\partial w}{\partial t}+\Delta w+\chi_{\omega} V\left(y-\tilde{\varphi}_{k}\right)=0 \text { in } \Omega \times(0, T) \\
w=0 \text { on } \Gamma \times(0, T) \\
w(0)=\left(y_{0}-\varphi_{k}\right)-P_{k} y_{0} \text { in } \Omega .
\end{array}\right.
$$

We have

$$
\|w\|_{C\left([0, T] ; H_{\Delta}(\Omega)\right)} \leq C\left(\left\|\left(y_{0}-\varphi_{k}\right)-P_{k} y_{0}\right\|_{H_{\Delta}(\Omega)}+\left\|V\left(y-\tilde{\varphi}_{k}\right)\right\|_{L^{2}\left(0, T ; H^{3}(\Omega) \cap H_{0}^{1}(\Omega)\right)}\right)
$$

But

$$
\left\|\left(y_{0}-\varphi_{k}\right)-P_{k} y_{0}\right\|_{H_{\Delta}(\Omega)} \leq C\left\|y_{0}-\varphi_{k}\right\|_{H_{\Delta}(\Omega)}^{2}
$$

and

$$
\begin{aligned}
& \left\|y-\tilde{\varphi}_{k}\right\|_{C\left([0, T] ; H_{\Delta}(\Omega)\right)} \leq C\left(\left\|y_{0}-\varphi_{k}\right\|_{H_{\Delta}(\Omega)}+\|V y\|_{L^{2}\left(0, T ; H^{3}(\Omega) \cap H_{0}^{1}(\Omega)\right)}\right) \\
& \leq C\left(\left\|y_{0}-\varphi_{k}\right\|_{H_{\Delta}(\Omega)}+C\|V\|_{C([0, T] ; E)}\left(\left\|y_{0}\right\|_{H_{\Delta}(\Omega)}+\|V\|_{C([0, T] ; E)}\right)\right) .
\end{aligned}
$$

Therefore

$$
\|w\|_{C\left([0, T] ; H_{\Delta}(\Omega)\right)} \leq C\left(\left\|y_{0}-\varphi_{k}\right\|_{H_{\Delta}(\Omega)}+C\|V\|_{C([0, T] ; E)}\right)^{2}
$$

and this proves the differentiability of $\Lambda$. To prove that $\Re\left(\tilde{z}(T), \tilde{\varphi}_{k}(T)\right)_{L^{2}(\Omega)}=0$ it suffices to notice that $\frac{\mathrm{d}}{\mathrm{d} t}\left(\tilde{z}(t), \tilde{\varphi}_{k}(t)\right)_{L^{2}(\Omega)}$ is purely imaginary. This finishes the proof of Lemma 3.4 .

Now we write

$$
z(t)=\mathrm{e}^{i \lambda_{k} t} \tilde{z}(t)
$$

and we have to show that $\Lambda^{\prime}$ has a right continuous inverse which means (using the reversibility of Schrödinger equation) that we have to solve a null controllability problem for the problem

$$
\left\{\begin{array}{l}
i \frac{\partial z}{\partial t}+\Delta z+\lambda_{k} z+\chi_{\omega} W \varphi_{k}=0 \text { in } \Omega \times(0, T), \\
z=0 \text { on } \Gamma \times(0, T), \\
z(0)=z_{0} \text { in } \Omega .
\end{array}\right.
$$

More precisely, we want to show that for any $z_{0} \in H_{\Delta}(\Omega) \cap T S_{k}$, we can find a real potential (control) $W \in C([0, T] ; E)$ such that the solution of (3.10) satisfies

$$
z(T)=0 .
$$

The following result has an independent interest and it follows essentially the lines of the analogous result in [3]. 
Proposition 3.5. Let $T>0$ and let us assume that $\omega$ is constructed as in (3.1) with $\Gamma_{0}$ satisfying (2.4). Then for every $z_{0} \in T S_{k}$, there exists a real control $g \in C\left([0, T] ; L^{2}(\Omega)\right)$ such that if $z$ is the solution of

$$
\left\{\begin{array}{l}
i \frac{\partial z}{\partial t}+\Delta z+\lambda_{k} z+\chi_{\omega} g=0 \text { in } \Omega \times(0, T), \\
z=0 \text { on } \Gamma \times(0, T) \\
z(0)=z_{0} \text { in } \Omega
\end{array}\right.
$$

then we have

$$
z(T)=0
$$

Proof. Let us consider the adjoint equation

$$
\left\{\begin{array}{l}
i \frac{\partial \psi}{\partial t}+\Delta \psi+\lambda_{k} \psi=0 \text { in } \Omega \times(0, T) \\
\psi=0 \text { on } \Gamma \times(0, T) \\
\psi(0)=\psi_{0} \text { in } \Omega
\end{array}\right.
$$

(notice that $\tilde{\psi}=\mathrm{e}^{-i \lambda_{k} t} \psi$ is solution of the free Schrödinger equation), and let us take $T_{0}$ such that $0<T_{0}<T$ and $\delta>0$ such that $4 \delta \leq\left(T-T_{0}\right)$.

From the construction of $\omega$ and of $\chi_{\omega}$, and the result by [9] already mentioned, we know that we have the internal observality inequality

$$
\left|\psi_{0}\right|_{L^{2}(\Omega)}^{2}=|\psi(2 \delta)|_{L^{2}(\Omega)}^{2} \leq C \int_{\omega_{\epsilon_{1} \times\left(2 \delta, T_{0}+2 \delta\right)}}|\psi|^{2} \mathrm{~d} x \mathrm{~d} t \leq C \int_{\omega \times\left(2 \delta, T_{0}+2 \delta\right)} \chi_{\omega}|\psi|^{2} \mathrm{~d} x \mathrm{~d} t .
$$

It is then well known from Lions' HUM (see [8]) that (3.14) implies for any $z_{0} \in L^{2}(\Omega)$ the existence of a complex valued control $g$ such that $z(T)=0$.

The difference here is that we require a real valued control.

Now we take $z_{0} \in T S_{k}$. Let $\psi$ (respectively $\hat{\psi}$ ) be the solution of (3.13) with initial value $\psi_{0} \in T S_{k}$ (respectively $\left.\hat{\psi}_{0} \in T S_{k}\right)$. We define a function $\eta \in C_{0}^{\infty}(\mathbb{R})$ such that

$$
\left\{\begin{array}{l}
0 \leq \eta(t) \leq 1, \forall t \in \mathbb{R} \\
\eta(t)=1, \forall t \in[2 \delta, T-2 \delta] \\
\operatorname{Supp}(\eta)=[\delta, T-\delta], \eta(t) \neq 0 \text { for } t \in(\delta, T-\delta)
\end{array}\right.
$$

This function $\eta$ will be required for the proof of Lemma 3.6 below and for the regularity of the control later on.

We define $w$ as the solution of the (backward) equation

$$
\left\{\begin{array}{l}
i \frac{\partial w}{\partial t}+\Delta w+\lambda_{k} w+\eta \chi_{\omega} \Im \psi=0 \text { in } \Omega \times(0, T), \\
w=0 \text { on } \Gamma \times(0, T), \\
w(T)=0 \text { in } \Omega .
\end{array}\right.
$$

As $\psi \in C\left([0, T] ; L^{2}(\Omega)\right)$ we also have $w \in C\left([0, T] ; L^{2}(\Omega)\right)$ so that $w(0) \in L^{2}(\Omega)$ and it can be easily shown that $w(0) \in T S_{k}$. Now multiplying (3.16) by $\hat{\psi}$ and integrating by parts, we obtain for every $\psi_{0}, \hat{\psi}_{0} \in T S_{k}$

$$
-i \int_{\Omega} w(0) \overline{\hat{\psi}}_{0} \mathrm{~d} x+\int_{\omega \times(0, T)} \eta \chi_{\omega} \Im \psi \overline{\hat{\psi}} \mathrm{d} x \mathrm{~d} t=0 .
$$

This implies

$$
-\Re\left(w(0), \hat{\psi}_{0}\right)_{L^{2}(\Omega)}=\int_{\omega \times(0, T)} \eta \chi_{\omega} \Im \psi \Im \hat{\psi} \mathrm{d} x \mathrm{~d} t .
$$

In order to solve our controllability problem, we would like to find $\psi_{0}$ such that $w(0)=z_{0}$ or equivalently such that

$$
\forall \hat{\psi}_{0} \in T S_{k}, \Re\left(w(0), \hat{\psi}_{0}\right)_{L^{2}(\Omega)}=\Re\left(z_{0}, \hat{\psi}_{0}\right)_{L^{2}(\Omega)} .
$$

Then the control $g=\eta \chi_{\omega} \Im \psi$ and the corresponding solution of (3.12) $z=w$ would solve our problem. 
Let us define

$$
\forall \psi_{0}, \hat{\psi}_{0} \in T S_{k}, a\left(\psi_{0}, \hat{\psi}_{0}\right)=\int_{\omega \times(0, T)} \eta \chi_{\omega} \Im \psi \Im \hat{\psi} \mathrm{d} x \mathrm{~d} t .
$$

We want to find a solution $\psi_{0} \in T S_{k}$ of the variational problem

$$
a\left(\psi_{0}, \hat{\psi}_{0}\right)=-\Re \int_{\Omega} z_{0} \overline{\hat{\psi}}_{0} \mathrm{~d} x, \forall \hat{\psi}_{0} \in T S_{k} .
$$

We know that $T S_{k}$, equipped with the scalar product $\Re(z, \hat{z})_{L^{2}(\Omega)}$ is a real Hilbert space, and it is clear that $a(.,$.$) is a bilinear continuous form on T S_{k} \times T S_{k}$. If we can prove a coercivity inequality of the form

$$
\exists C>0, \forall \psi_{0} \in T S_{k},\left|\psi_{0}\right|_{L^{2}(\Omega)}^{2} \leq C a\left(\psi_{0}, \psi_{0}\right)=C \int_{\omega \times(0, T)} \eta \chi_{\omega}|\Im \psi|^{2} \mathrm{~d} x \mathrm{~d} t,
$$

then by Lax-Milgram theorem, (3.18) will have a unique solution $\psi_{0}$ and this will prove Proposition 3.5.

Notice that (3.19) is another type of internal observability inequality and we will prove it in two steps which follow the lines of [3].

Lemma 3.6. There exists $C>0$ such that for every $\psi_{0} \in L^{2}(\Omega)$

$$
\left|\psi_{0}\right|_{L^{2}(\Omega)}^{2} \leq C \int_{\omega \times(0, T)} \eta \chi_{\omega}|\Im \psi|^{2} \mathrm{~d} x \mathrm{~d} t+C|| \psi_{0} \|_{H^{-2}(\Omega)}^{2}
$$

Proof. We have

$$
2 \Im \psi=\frac{\psi-\bar{\psi}}{i}
$$

so that

$$
4|\Im \psi|^{2}=-(\psi)^{2}-(\bar{\psi})^{2}+2|\psi|^{2}
$$

and

$$
\left.|\psi|^{2}=2|\Im \Psi|^{2}+\frac{1}{2}\left((\psi)^{2}\right)+(\bar{\psi})^{2}\right) .
$$

From the internal observability inequality (3.14) we have

$$
\begin{aligned}
\left|\psi_{0}\right|_{L^{2}(\Omega)}^{2} & =|\psi(2 \delta)|_{L^{2}(\Omega)}^{2} \leq C \int_{\omega \times\left(2 \delta, T_{0}+2 \delta\right)} \chi_{\omega}|\psi|^{2} \mathrm{~d} x \mathrm{~d} t \leq C \int_{\omega \times(0, T)} \eta \chi_{\omega}|\psi|^{2} \mathrm{~d} x \mathrm{~d} t \\
& \leq 2 C \int_{\omega \times(0, T)} \eta \chi_{\omega}|\Im \psi|^{2} \mathrm{~d} x \mathrm{~d} t+\frac{C}{2}\left|\int_{\omega \times(0, T)} \eta \chi_{\omega}(\psi)^{2} \mathrm{~d} x \mathrm{~d} t\right|+\frac{C}{2}\left|\int_{\omega \times(0, T)} \eta \chi_{\omega}(\bar{\psi})^{2} \mathrm{~d} x \mathrm{~d} t\right| .
\end{aligned}
$$

Let us show that

$$
\left|\int_{\omega \times(0, T)} \eta \chi_{\omega}(\psi)^{2} \mathrm{~d} x \mathrm{~d} t\right| \leq C\left\|\psi_{0}\right\|_{H^{-2}(\Omega)} .
$$

In the same way we will show that

$$
\left|\int_{\omega \times(0, T)} \eta \chi_{\omega}(\bar{\psi})^{2} \mathrm{~d} x \mathrm{~d} t\right| \leq C\left\|\psi_{0}\right\|_{H^{-2}(\Omega)} .
$$

We can decompose $\psi_{0}$ on the hilbert basis of eigenfunctions

$$
\psi_{0}=\sum_{j=1}^{+\infty} a_{j} \varphi_{j}
$$


with

$$
\sum_{j=1}^{+\infty}\left|a_{j}\right|^{2}=\left|\psi_{0}\right|_{L^{2}(\Omega)}^{2} \text { and } \sum_{j=1}^{+\infty} \frac{\left|a_{j}\right|^{2}}{\lambda_{j}^{2}}=\left\|\psi_{0}\right\|_{H^{-2}(\Omega)}^{2} .
$$

Then

$$
\psi(t)=\sum_{j=1}^{+\infty} a_{j}(t) \varphi_{j}
$$

where

$$
i a_{j}^{\prime}(t)-\lambda_{j} a_{j}(t)+\lambda_{k} a_{j}(t)=0, \text { and } a_{j}(0)=a_{j},
$$

so that

$$
\psi(t)=\sum_{j=1}^{+\infty} a_{j} \mathrm{e}^{-i\left(\lambda_{j}-\lambda_{k}\right) t} \varphi_{j}
$$

Therefore

$$
\begin{aligned}
\int_{\omega \times(0, T)} \eta \chi_{\omega}(\psi)^{2} \mathrm{~d} x \mathrm{~d} t & =\sum_{j, l=1}^{+\infty} \int_{\omega \times(0, T)}\left(\eta \mathrm{e}^{2 i \lambda_{k} t}\right) \mathrm{e}^{-i\left(\lambda_{j}+\lambda_{l}\right) t} a_{j} a_{l} \varphi_{j} \varphi_{l} \mathrm{~d} x \mathrm{~d} t \\
& =\sum_{j, l=1}^{+\infty} \int_{\omega} a_{j} a_{l} \varphi_{j} \varphi_{l} \mathrm{~d} x \int_{0}^{T}\left(\eta \mathrm{e}^{2 i \lambda_{k} t}\right) \mathrm{e}^{-i\left(\lambda_{j}+\lambda_{l}\right) t} \mathrm{~d} t .
\end{aligned}
$$

Now we can integrate by parts $2 m$ times the term $\int_{0}^{T}\left(\eta \mathrm{e}^{2 i \lambda_{k} t}\right) \mathrm{e}^{-i\left(\lambda_{j}+\lambda_{l}\right) t} \mathrm{~d} t$ to obtain

$$
\left|\int_{0}^{T}\left(\eta \mathrm{e}^{2 i \lambda_{k} t}\right) \mathrm{e}^{-i\left(\lambda_{j}+\lambda_{l}\right) t} \mathrm{~d} t\right| \leq \frac{C}{\left(\lambda_{j}+\lambda_{l}\right)^{2 m}}
$$

where $C$ depends on $\lambda_{k}$ and on derivatives of $\eta$.

We then obtain

$$
\begin{aligned}
\left|\int_{\omega \times(0, T)} \eta \chi_{\omega}(\psi)^{2} \mathrm{~d} x \mathrm{~d} t\right| & \leq C \sum_{j, l=1}^{+\infty} \int_{\Omega} \frac{\left|a_{j}\right|\left|a_{l}\right|}{\left(\lambda_{j}+\lambda_{l}\right)^{2 m}}\left|\varphi_{j}\right|\left|\varphi_{l}\right| \mathrm{d} x \\
& \leq C \sum_{j, l=1}^{+\infty} \frac{\left|a_{j}\right|}{\lambda_{j}^{m}} \frac{\left|a_{l}\right|}{\lambda_{l}^{m}} \leq C\left(\sum_{j=1}^{+\infty} \frac{\left|a_{j}\right|}{\lambda_{j}^{m}}\right)^{2} \\
& \leq C\left(\sum_{j=1}^{+\infty} \frac{\left|a_{j}\right|^{2}}{\lambda_{j}^{2}}\right)\left(\sum_{j=1}^{+\infty} \frac{1}{\lambda_{j}^{(2 m-2)}}\right) \\
& \leq C|| \psi_{0} \|_{H^{-2}(\Omega)}
\end{aligned}
$$

from Weyl's theorem [12] if we choose $m$ large enough.

This proves Lemma 3.6.

Lemma 3.7. There exists $C>0$ such that for every $\psi_{0} \in T S_{k}$,

$$
\left|\psi_{0}\right|_{L^{2}(\Omega)} \leq C \int_{\omega \times(0, T)} \eta \chi_{\omega}|\Im \psi|^{2} \mathrm{~d} x \mathrm{~d} t=C a\left(\psi_{0}, \psi_{0}\right) .
$$


Proof. We already know from Lemma 3.6 that there exists $C>0$ such that for $\psi_{0} \in L^{2}(\Omega)$

$$
C a\left(\psi_{0}, \psi_{0}\right)=\left.C \int_{\omega \times(0, T)} \eta \chi_{\omega}|\Im \psi|^{2}|\mathrm{~d} x \mathrm{~d} t \geq| \psi_{0}\right|_{L^{2}(\Omega)}-C\left\|\psi_{0}\right\|_{H^{-2}(\Omega)}
$$

and we know that $L^{2}(\Omega)$ is compactly embedded in $H^{-2}(\Omega)$. It is then standard to show that if we have the uniqueness property

$$
\psi_{0} \in T S_{k} \text { and } a\left(\psi_{0}, \psi_{0}\right)=0 \Longrightarrow \psi_{0}=0
$$

then there exists $C>0$ such that

$$
\forall \psi_{0} \in T S_{k}, C a\left(\psi_{0}, \psi_{0}\right) \geq\left|\psi_{0}\right|_{L^{2}(\Omega)} .
$$

We then have to prove the uniqueness property. Let

$$
K=\left\{\psi_{0} \in T S_{k}, a\left(\psi_{0}, \psi_{0}\right)=0\right\} .
$$

If $\psi_{0} \in K$, it says that

$$
\left\{\begin{array}{l}
\psi_{0} \in T S_{k} \\
i \frac{\partial \psi}{\partial t}+\Delta \psi+\lambda_{k} \psi=0 \text { in } \Omega \times(0, T) \\
\psi=0 \text { on } \Gamma \times(0, T) \\
\psi(0)=\psi_{0} \text { in } \Omega
\end{array}\right.
$$

and $\eta \chi_{\omega} \Im \psi=0$ on $(0, T)$ which implies $\chi_{\omega} \Im \psi=0$ on $(\delta, T-\delta)$.

Then $K$ is a (real) vector subspace of $L^{2}(\Omega)$ and from Lemma 3.6 we know that

$$
\forall \psi_{0} \in K,\left|\psi_{0}\right|_{L^{2}(\Omega)} \leq C|| \psi_{0} \|_{H^{-2}(\Omega)} .
$$

Because of the compact embedding of $L^{2}(\Omega)$ in $H^{-2}(\Omega)$ we immediately see that $K$ must be of finite dimension $J$. Let us take $\tau$ such that $0<\tau<\frac{\delta}{2}$ and define

$$
\psi_{\tau}(t)=\frac{\psi(t+\tau)-\psi(t)}{\tau}
$$

Then $\chi_{\omega} \Im \psi_{\tau}=0$ on $(\delta, T-\tau-\delta) \supset\left(\delta, T-\frac{\delta}{2}\right)$.

Notice that Lemma 3.6 is still valid if we change the function $\eta$ in $\tilde{\eta}$ such that $\operatorname{Supp} \tilde{\eta}=\left[\delta, T-3 \frac{\delta}{2}\right]$ and $\tilde{\eta} \neq 0$ on $\left(\delta, T-3 \frac{\delta}{2}\right)$.

As $\chi_{\omega} \Im \psi_{\tau}=0$ on $\left(\delta, T-3 \frac{\delta}{2}\right)$ we still have

$$
\forall \tau, 0<\tau<\frac{\delta}{2},\left|\psi_{\tau}(0)\right|_{L^{2}(\Omega)} \leq C|| \psi_{\tau}(0) \|_{H^{-2}(\Omega)} .
$$

But $\psi_{\tau}(0)$ converges to $\frac{\partial \psi}{\partial t}(0)=i\left(\Delta \psi_{0}+\lambda_{k} \psi_{0}\right)$ in $H^{-2}(\Omega)$ when $\tau$ tends to 0 .

From the above inequality we see that in fact

$$
\frac{\partial \psi}{\partial t}(0)=i\left(\Delta \psi_{0}+\lambda_{k} \psi_{0}\right) \in L^{2}(\Omega)
$$

On the other hand, as $\psi_{0} \in T S_{k}$ we have $\psi(t) \in T S_{k}$ for $t \in(0, T)$ so that $\psi_{\tau}(0) \in T S_{k}$ which implies $\frac{\partial \psi}{\partial t}(0)=i\left(\Delta \psi_{0}+\lambda_{k} \psi_{0}\right)=\hat{\psi}_{0} \in T S_{k}$. 
Let $\hat{\psi}$ be the solution of (3.13) with initial value $\hat{\psi}_{0}$. We know that $\hat{\psi}(t)=\lim _{\tau \rightarrow 0} \psi_{\tau}(t)$.

We know that $\chi_{\omega} \Im \psi_{\tau}=0$ on $[\delta, T-\delta-\tau]$ so that $\chi_{\omega} \Im \hat{\psi}=0$ on $(\delta, T-\delta)$ and $\eta \chi_{\omega} \Im \hat{\psi}=0$ on $(0, T)$.

Therefore $\hat{\psi}_{0} \in K$ and $i\left(\Delta \psi_{0}+\lambda_{k} \psi_{0}\right) \in K$.

Now the operator $-i\left(\Delta+\lambda_{k} I\right)$ maps $K$ into $K$, is antisymmetric, and $K$ is finite dimensional.

If $K \neq\{0\}$, we can diagonalize this operator on $K$ and obtain an orthonormal family of eigenfunctions $\left(w_{1}, \ldots, w_{J}\right)$ with $J=\operatorname{dim} K$ and corresponding purely imaginary eigenvalues $\left(i \mu_{1}, \ldots, i \mu_{J}\right)$. For $j=1, \ldots, J$ we write $\tilde{w}_{j}$ the solution of

$$
\left\{\begin{array}{l}
i \frac{\partial \tilde{w}_{j}}{\partial t}+\Delta \tilde{w}_{j}+\lambda_{k} \tilde{w}_{j}=0 \text { in } \Omega \times(0, T), \\
\tilde{w}_{j}=0 \text { on } \Gamma \times(0, T), \\
\tilde{w}_{j}(0)=w_{j} \text { in } \Omega,
\end{array}\right.
$$

We know, because $w_{j} \in K$, that $w_{j} \in T S_{k}$ and $\chi_{\omega} \Im \tilde{w}_{j}=0$ on $(\delta, T-\delta)$. But also $-\Delta w_{j}-\lambda_{k} w_{j}=\mu_{j} w_{j}$ so that $w_{j}$ is very regular and therefore $\left(\mu_{j}+\lambda_{k}\right)$ is a real eigenvalue $\lambda_{l}$ of the Laplace operator with Dirichlet boundary conditions. Now we have

$$
\tilde{w}_{j}(t)=\mathrm{e}^{-i \mu_{j} t} w_{j} \text {, and } \chi_{\omega} \Im \tilde{w}_{j}=0 \text { on }(\delta, T-\delta) .
$$

If $\mu_{j} \neq 0$ this implies $\chi_{\omega} \Im w_{j}=\chi_{\omega} \Re w_{j}=0$ in $\Omega$ and as $w_{j}$ is an eigenfunction of $-\Delta$ this implies $w_{j}=0$ which is impossible.

If $\mu_{j}=0$, because $\lambda_{k}$ is a simple eigenvalue, we have $w_{j}=\mu \varphi_{k}$ with $\mu \in \mathbb{C},|\mu|=1$, and as $w_{j} \in T S_{k}$ this implies $\mu= \pm i$ but as $\Im w_{j}=0$ we have $w_{j}=0$ which is impossible.

Therefore we have a contradiction and $K$ must be reduced to $\{0\}$. This proves the uniqueness property and finishes the proof of Lemma 3.7 and of Proposition 3.5.

We now want to prove that when the initial condition $z_{0}$ is element of $H_{\Delta}(\Omega) \cap T S_{k}$ we can choose a real control $\eta \chi_{\omega} g$ in a more regular space, namely in $C\left([0, T] ; H^{3}(\Omega) \cap H_{0}^{1}(\Omega)\right)$. We denote by $A$ the operator

$$
A=-\left(\Delta+\lambda_{k} I\right) \text {. }
$$

with Dirichlet boundary conditions. Notice that $D(A)=H^{2}(\Omega) \cap H_{0}^{1}(\Omega)$ and $D\left(A^{\frac{3}{2}}\right)=H_{\Delta}(\Omega)$.

Lemma 3.8. If $z_{0} \in H_{\Delta}(\Omega) \cap T S_{k}$, then the solution $\psi_{0}$ of (3.18) satisfies

$$
\psi_{0} \in H_{\Delta}(\Omega) \cap T S_{k} .
$$

This implies that $\eta \chi_{\omega} \Im \psi \in C\left([0, T] ; H^{3}(\Omega) \cap H_{0}^{1}(\Omega)\right)$.

Proof. We adapt slightly here the argument of [5] which can be almost followed term by term but for sake of completeness we give the complete proof here.

We recall that $\psi_{0} \in T S_{k}$ is solution of

$$
a\left(\psi_{0}, \hat{\psi}_{0}\right)=\int_{\omega \times(0, T)} \eta \chi_{\omega} \Im \psi \Im \hat{\psi} \mathrm{d} x \mathrm{~d} t=-\Re \int_{\Omega} z_{0} \overline{\hat{\psi}}_{0} \mathrm{~d} x, \forall \hat{\psi}_{0} \in T S_{k}
$$

We assume now that $z_{0} \in D(A)$. Let $\tau$ be such that $0<\tau<\delta$. As the Schrödinger equation corresponds to a group we can extend all equations on $(-\delta, T+\delta)$ and we recall that in the equation for $z$ we have $\operatorname{Supp}(\eta)=[\delta, T-\delta]$. Let us define

$$
\hat{\psi}_{0}=\frac{\psi(\tau)-2 \psi_{0}+\psi(-\tau)}{\tau^{2}} .
$$

Then

$$
\hat{\psi}(t)=\frac{\psi(t+\tau)-2 \psi(t)+\psi(t-\tau)}{\tau^{2}} .
$$


Let $H$ be defined by

$$
H=\int_{\omega \times(0, T)} \eta \chi_{\omega} \Im \psi \Im \hat{\psi} \mathrm{d} x \mathrm{~d} t=\int_{\omega \times(0, T)} \eta(t) \chi_{\omega} \Im \psi(t) \Im\left(\frac{\psi(t+\tau)-2 \psi(t)+\psi(t-\tau)}{\tau^{2}}\right) \mathrm{d} x \mathrm{~d} t
$$

On the one hand we have

$$
H=-\Re\left(z_{0}, \frac{\psi(\tau)-2 \psi_{0}+\psi(-\tau)}{\tau^{2}}\right)_{L^{2}(\Omega)} .
$$

On the other hand, because $\eta$ vanishes outside $(\delta, T-\delta)$, some elementary computations give

$$
\begin{aligned}
& H=\frac{1}{\tau} \int_{\omega \times(0, T)} \eta(t) \chi_{\omega} \Im \psi(t) \Im\left(\frac{\psi(t+\tau)-\psi(t)}{\tau}\right) \mathrm{d} x \mathrm{~d} t \\
& -\frac{1}{\tau} \int_{\omega \times(0, T)} \eta(t) \chi_{\omega} \Im \psi(t) \Im\left(\frac{\psi(t)-\psi(t-\tau)}{\tau}\right) \mathrm{d} x \mathrm{~d} t \\
& =\frac{1}{\tau} \int_{\omega \times(-\tau, T)} \eta(t) \chi_{\omega} \Im \psi(t) \Im\left(\frac{\psi(t+\tau)-\psi(t)}{\tau}\right) \mathrm{d} x \mathrm{~d} t \\
& -\frac{1}{\tau} \int_{\omega \times(-\tau, T)} \eta(t+\tau) \chi_{\omega} \Im \psi(t+\tau) \Im\left(\frac{\psi(t+\tau)-\psi(t)}{\tau}\right) \mathrm{d} x \mathrm{~d} t \\
& =\int_{\omega \times(-\tau, T)}\left(\frac{\eta(t)-\eta(t+\tau)}{\tau}\right) \chi_{\omega} \Im\left(\frac{\psi(t)+\psi(t+\tau)}{2}\right) \Im\left(\frac{\psi(t+\tau)-\psi(t)}{\tau}\right) \mathrm{d} x \mathrm{~d} t \\
& +\int_{\omega \times(-\tau, T)}\left(\frac{\eta(t)+\eta(t+\tau)}{\tau}\right) \chi_{\omega} \Im\left(\frac{\psi(t)-\psi(t+\tau)}{2}\right) \Im\left(\frac{\psi(t+\tau)-\psi(t)}{\tau}\right) \mathrm{d} x \mathrm{~d} t .
\end{aligned}
$$

Notice that all integrals between $-\tau$ and 0 vanish because of the presence of $\eta$. We know that

$$
\left|\frac{\eta(t)-\eta(t+\tau)}{\tau}\right| \leq\left\|\eta^{\prime}\right\|_{L^{\infty}(0 . T)}
$$

and because of the equation (3.18) satisfied by $\psi_{0}$ and the coercivity condition (3.19)

$$
\left|\psi_{0}\right|_{L^{2}(\Omega)} \leq C\left|z_{0}\right|_{L^{2}(\Omega)} .
$$

Therefore, from the preservation of the $L^{2}$ norm by the Schrödinger equation, we obtain

$$
\begin{gathered}
H \leq C \int_{\omega \times(0, T)} \chi_{\omega}|\psi(t)+\psi(t+\tau)|\left|\frac{\psi(t+\tau)-\psi(t)}{\tau}\right| \mathrm{d} x \mathrm{~d} t \\
-\frac{1}{2} \int_{\omega \times(0, T)}(\eta(t+\tau)+\eta(t)) \chi_{\omega}\left|\Im\left(\frac{\psi(t+\tau)-\psi(t))}{\tau}\right)\right|^{2} \mathrm{~d} x \mathrm{~d} t \\
\leq C\left|z_{0}\right|_{L^{2}(\Omega)}\left|\frac{\psi(\tau)-\psi_{0}}{\tau}\right|_{L^{2}(\Omega)}-\frac{1}{2} \int_{\omega \times(0, T)}(\eta(t+\tau)+\eta(t)) \chi_{\omega}\left|\Im\left(\frac{\psi(t+\tau)-\psi(t))}{\tau}\right)\right|^{2} \mathrm{~d} x \mathrm{~d} t
\end{gathered}
$$

Now from the observability inequality obtained in Lemma 3.7 we have

$$
\begin{aligned}
\left|\frac{\psi(\tau)-\psi_{0}}{\tau}\right|_{L^{2}(\Omega)}^{2} & \leq \frac{C}{2} \int_{\omega \times(0, T)} \eta(t) \chi_{\omega}\left|\Im\left(\frac{\psi(t+\tau)-\psi(t))}{\tau}\right)\right|^{2} \mathrm{~d} x \mathrm{~d} t \\
& \leq \frac{C}{2} \int_{\omega \times(0, T)}(\eta(t+\tau)+\eta(t)) \chi_{\omega}\left|\Im\left(\frac{\psi(t+\tau)-\psi(t))}{\tau}\right)\right|^{2} \mathrm{~d} x \mathrm{~d} t .
\end{aligned}
$$


Therefore

$$
\left|\frac{\psi(\tau)-\psi_{0}}{\tau}\right|_{L^{2}(\Omega)}^{2} \leq C\left|z_{0}\right|_{L^{2}(\Omega)}\left|\frac{\psi(\tau)-\psi_{0}}{\tau}\right|_{L^{2}(\Omega)}-C H
$$

From (3.23) we have

$$
H=\Re\left(z_{0}, \frac{\psi(\tau)-2 \psi_{0}+\psi(-\tau)}{\tau^{2}}\right)_{L^{2}(\Omega)}=\Re\left(z_{0}, \frac{\psi(\tau)-\psi_{0}}{\tau^{2}}\right)_{L^{2}(\Omega)}-\Re\left(z_{0}, \frac{\psi_{0}-\psi(-\tau)}{\tau^{2}}\right)_{L^{2}(\Omega)} .
$$

Because $\eta$ vanishes on $(0, \tau)$ the function $z$ satisfies

$$
\left\{\begin{array}{l}
i \frac{\partial z}{\partial t}+\Delta z+\lambda_{k} z=0 \text { in } \Omega \times(0, \tau) \\
z=0 \text { on } \Gamma \times(0, \tau) \\
z(0)=z_{0} \text { in } \Omega
\end{array}\right.
$$

If we define

$$
\psi_{-\tau}(t)=\left(\frac{\psi(t-\tau)-\psi(t)}{\tau}\right)
$$

this function satisfies

$$
\left\{\begin{array}{l}
i \frac{\partial \psi_{-\tau}}{\partial t}+\Delta \psi_{-\tau}+\lambda_{k} \psi_{-\tau}=0 \text { in } \Omega \times(0, \tau), \\
\psi_{-\tau}=0 \text { on } \Gamma \times(0, \tau), \\
\psi_{-\tau}(0)=\left(\frac{\psi_{-\tau}-\psi_{0}}{\tau}\right) \text { in } \Omega .
\end{array}\right.
$$

Then, multiplying the equation for $z$ by $\psi_{-\tau}$ and integrating on $(0, \tau)$ gives

$$
i\left(z(\tau),\left(\frac{\psi_{0}-\psi(\tau)}{\tau^{2}}\right)\right)_{L^{2}(\Omega)}-i\left(z_{0},\left(\frac{\psi(-\tau)-\psi_{0}}{\tau^{2}}\right)\right)_{L^{2}(\Omega)}=0 .
$$

Therefore we have

$$
\begin{array}{r}
H=\Re\left(z_{0}, \frac{\psi(\tau)-\psi_{0}}{\tau^{2}}\right)_{L^{2}(\Omega)}-\Re\left(z(\tau),\left(\frac{\psi(\tau)-\psi(0)}{\tau^{2}}\right)\right)_{L^{2}(\Omega)} \\
=\Re\left(\left(\frac{z_{0}-z(\tau)}{\tau}\right),\left(\frac{\psi(\tau)-\psi_{0}}{\tau}\right)\right)_{L^{2}(\Omega)}
\end{array}
$$

Then for $\tau$ small enough we obtain

$$
\begin{aligned}
|H| \leq & \left|\frac{z_{0}-z(\tau)}{\tau}\right|_{L^{2}(\Omega)}\left|\frac{\psi(\tau)-\psi_{0}}{\tau}\right|_{L^{2}(\Omega)} \\
& \leq C\left|A z_{0}\right|_{L^{2}(\Omega)}\left|\frac{\psi(\tau)-\psi_{0}}{\tau}\right|_{L^{2}(\Omega)}
\end{aligned}
$$

Using (3.24) we obtain

$$
\left|\frac{\psi(\tau)-\psi_{0}}{\tau}\right|_{L^{2}(\Omega)}^{2} \leq C\left|z_{0}\right|_{L^{2}(\Omega)}\left|\frac{\psi(\tau)-\psi_{0}}{\tau}\right|_{L^{2}(\Omega)}+C\left|A z_{0}\right|_{L^{2}(\Omega)}\left|\frac{\psi(\tau)-\psi_{0}}{\tau}\right|_{L^{2}(\Omega)}
$$

and therefore

$$
\forall \tau>0 \text { small enough, }\left|\frac{\psi(\tau)-\psi_{0}}{\tau}\right|_{L^{2}(\Omega)}^{2} \leq C\left\|z_{0}\right\|_{D(A)}^{2} .
$$


This implies that $A \psi_{0} \in L^{2}(\Omega)$ with $\left|A \psi_{0}\right|_{L^{2}(\Omega)} \leq C\left\|z_{0}\right\|_{D(A)}$ which means

$$
\psi_{0} \in D(A) \text { and }\left\|\psi_{0}\right\|_{D(A)} \leq C\left\|z_{0}\right\|_{D(A)} .
$$

We can iterate this process exactly in the same way to show that when $z_{0} \in D\left(A^{2}\right) \cap T S_{k}$, then $\psi_{0} \in D\left(A^{2}\right)$ and

$$
\left\|\psi_{0}\right\|_{D\left(A^{2}\right)} \leq C\left\|z_{0}\right\|_{D\left(A^{2}\right)} .
$$

By interpolation we see that when $z_{0} \in D\left(A^{\frac{3}{2}}\right) \cap T S_{k}$ then $\psi_{0} \in D\left(A^{\frac{3}{2}}\right)$ and

$$
\left\|\psi_{0}\right\|_{D\left(A^{\frac{3}{2}}\right)} \leq C\left\|z_{0}\right\|_{D\left(A^{\frac{3}{2}}\right)} .
$$

As $H_{\Delta}(\Omega)=D\left(A^{\frac{3}{2}}\right)$ we see that $z_{0} \in H_{\Delta}(\Omega) \cap T S_{k}$ implies $\psi_{0} \in H_{\Delta}(\Omega)$ which itself implies $\psi \in$ $C\left([0, T] ; H_{\Delta}(\Omega)\right)$ and therefore $\eta \chi_{\omega} \Im \psi \in C\left([0, T] ; H^{3}(\Omega) \cap H_{0}^{1}(\Omega)\right)$.

This finishes the Proof of Lemma 3.8.

We have now proved that when the initial data $z_{0}$ satisfies $z_{0} \in H_{\Delta}(\Omega) \cap T S_{k}$, we can choose a real control $\eta \chi_{\omega} g \in C\left([0, T] ; H^{3}(\Omega) \cap H_{0}^{1}(\Omega)\right)$ in Proposition 3.5. This enables us to write this control in the form

$$
\eta \chi_{\omega} g=\eta \chi_{\omega} W \varphi_{k}, \text { with } W \in C([0, T] ; E) .
$$

All our constructions are continuous so that this proves that the derivative of the mapping $\Lambda$ defined in (3.5) at the point $\left(\varphi_{k}, 0\right)$ has a continuous right inverse.

Using an inverse mapping theorem, we can find a neighborhood $\mathcal{U}_{0}$ of 0 in $C([0, T] ; E)$ and a neighborhood $\mathcal{U}_{1}$ of $\left(\mathrm{e}^{-i \lambda T} \varphi_{k}, \varphi_{k}\right)$ in $H_{\Delta}(\Omega)^{2}$ such that for any $\left(y_{0}, y_{1}\right) \in \mathcal{U}_{1}$, there exists $V \in \mathcal{U}_{0}$ such that the solution $y$ of

$$
\left\{\begin{array}{l}
i \frac{\partial y}{\partial t}+\Delta y+\eta \chi_{\omega} V y=0 \text { in } \Omega \times(0, T) \\
y=0 \text { on } \Gamma \times(0, T) \\
y(0)=y_{0} \text { in } \Omega
\end{array}\right.
$$

satisfies

$$
y(T)=y_{1} .
$$

This finishes the Proof of Theorem 2.1.

\subsection{Proof of Theorem 2.2}

For the case of a rectangle in dimension $N=2$ or a fortiori for the case of an interval in dimension $N=1$ we consider the eigenfunction $\varphi_{k}$ and we take two non empty open subsets of $\Omega, \omega_{0}$ and $\omega$ such that

$$
\bar{\omega}_{0} \subset \omega
$$

and such that

$$
\varphi_{k} \neq 0 \text { in } \bar{\omega}
$$

Then we define $\chi_{\omega} \in C_{0}^{\infty}(\omega)$ such that

$$
\left\{\begin{array}{l}
0 \leq \chi_{\omega} \leq 1 \text { in } \omega \\
\chi_{\omega}=1 \text { on } \omega_{0}
\end{array}\right.
$$

Then we know from [6] that we have an observability inequality of the form

$$
\left|\psi_{0}\right|_{L^{2}(\Omega)} \leq C \int_{\omega_{0} \times(0, T)}|\psi|^{2} \mathrm{~d} x \mathrm{~d} t \leq C \int_{\omega \times(0, T)} \chi_{\omega}|\psi|^{2} \mathrm{~d} x \mathrm{~d} t .
$$

The rest of the proof is completely identical to the one for Theorem 2.1. We don't have to use here the regularity property given by [10] because the term $V y$ vanishes near the boundary of $\Omega$.

This finishes the Proof of Theorem 2.2. 
Acknowledgements. The author wants to thank K. Beauchard, C. Laurent and S. Ervedoza for fruitful and very helpful discussions. He also wants to thank the referees for their very careful work, for their suggestions and for pointing out some errors in the preliminary version of this article. This work was partially supported by the Agence Nationale de la Recherche (ANR), Projet Blanc EMAQS No. ANR-2011-BS01-017-01

\section{REFERENCES}

[1] K. Beauchard, Local controllability of a 1D Schrödinger equation. J. Math. Pures Appl. 84 (2005) 851-956.

[2] K. Beauchard and C. Laurent, Local controllability of 1D linear and nonlinear Schrödinger equations. J. Math. Pures Appl. 94 (2010) 520-554.

[3] K. Beauchard and C. Laurent, Local exact controllability of the 2D Schrödinger-Poisson system. Preprint hal-01333627 (2016).

[4] J. Ball, J. Marsden and M. Slemrod, Controllability for distributed bilinear systems. SIAM J. Cont. Optim. 20 (1982) 575-597.

[5] S. Ervedoza and E. Zuazua, A systematic method for building smooth controls for smooth data. Discrete Contin. Dyn. Syst. Ser. B 14 (2010) 1375-1401.

[6] S. Jaffard, Contrôle interne exact des vibrations d'une plaque rectangulaire. Port. Math. 47 (1990) 423-429.

[7] G. Lebeau, Contrôle de l'equation de Schrödinger. J. Math. Pures Appl. 71 (1992) 267-291.

[8] J.-L. Lions, Contrôlabilité exacte, perturbations et stabilization des systèmes distribués. Tome 1, Contrôlabilité exacte. Collection R.M.A 8, Masson (1988).

[9] E. Machtyngier, Exact controllability for the Schrödinger equation. SIAM J. Control Optim. 32 (1994) 24-34.

[10] J.-P. Puel, A regularity property for Schrödinger equations on bounded domains. Rev. Mat. Complut. 26 (2013) $183-192$.

[11] G. Tenenbaum, M. Tucsnak, Fast and strongly localized observation for the Schrödinger equation. Trans. Amer. Math. Soc. 361 (2009) 951-977.

[12] H. Weyl, Das asymptotisch Verteilungsgezetz der Eigenwerte linearer partieller Differentialgleichungen. Math. Ann. 71 (1912) $441-479$. 